\title{
Basilar Artery Plaque and Pontine Infarction Location and Vascular Geometry
}

\author{
Bum Joon Kim, ${ }^{a}$ Kyung Mi Lee, ${ }^{\mathrm{b}}$ Hyun Young Kim, ${ }^{\mathrm{c}}$ Young Seo Kim, ${ }^{\mathrm{c}}$ Seong-Ho Koh, ${ }^{\mathrm{d}}$ Sung Hyuk Heo, ${ }^{\mathrm{a}}$ \\ Dae-Il Chang ${ }^{\mathrm{a}}$ \\ ${ }^{a}$ Department of Neurology, Kyung Hee University Hospital, Kyung Hee University School of Medicine, Seoul, Korea \\ 'Department of Radiology, Kyung Hee University Hospital, Kyung Hee University School of Medicine, Seoul, Korea \\ 'Department of Neurology, Hanyang University Seoul Hospital, Hanyang University College of Medicine, Seoul, Korea \\ ${ }^{d}$ Department of Neurology, Hanyang University Guri Hospital, Hanyang University College of Medicine, Seoul, Korea
}

Background and Purpose Subclinical atherosclerotic plaques are common in patients with pontine infarctions (PIs) but without basilar artery (BA) stenosis. We hypothesized that BA plaque locations may differ by PI type and vertical location as well as vertebrobasilar artery geometry.

Methods Ninety-six patients with PI but without BA stenosis on magnetic resonance imaging (MRI) and magnetic resonance angiography were enrolled. Pls were classified by type (paramedian, deep, or lateral) and vertical location (rostral, middle, or caudal). Patients underwent highresolution MRI to evaluate BA plaque location (anterior, posterior, or lateral). The mid-BA angle on anteroposterior view and angle between the BA and dominant vertebral artery (BA-VA angle) on lateral view were measured.

Results The Pls were paramedian (72.9\%), deep (17.7\%), and lateral (9.4\%) type with a rostral (32.3\%), middle (42.7\%), and caudal (25.0\%) vertical location. The BA plaque locations differed by PI type $(P=0.03)$ and vertical location $(P<0.001)$; BA plaques were most frequent at the posterior wall in paramedian (37.1\%) and caudal (58.3\%) Pls and at the lateral wall in lateral (55.5\%) and middle (34.1\%) PIs. The BA-VA and mid-BA angles differed by BA plaque and PI vertical location; the greatest $B A-V A$ angle was observed in patients with posterior plaques $(P<0.001)$ and caudal PIs $(P<0.001)$. Greatest mid-BA angles were observed with lateral $B A$ plaques $(P=0.03)$ and middlelocated Pls $(P=0.03)$.

Conclusions Greater mid-BA angulation may enhance lateral plaque formation, causing lateral and middle Pls, whereas greater BA-VA angulation may enhance posterior plaque formation, causing paramedian or caudal PIs.

Keywords Brain stem infarctions; Basilar artery; Plaque, atherosclerotic; Hemodynamics; Magnetic resonance angiography
Correspondence: Dae-II Chang Department of Neurology, Kyung Hee University Hospital, Kyung Hee University School of Medicine, 23 Kyungheedae-ro, Dongdaemun-gu, Seoul 02447, Korea Tel: +82-2-958-8499 Fax: +82-2-958-8490 E-mail:dichang@khmc.or.kr

Received: April 22, 2017

Revised: August 27, 2017

Accepted: September 8, 2017

\section{Introduction}

Pontine infarctions (PIs) present diverse clinical syndromes by location. ' Caudal Pls are more frequently present as severe uni- lateral weakness, whereas rostral Pls tend to be associated with ataxic symptoms. ${ }^{2}$ Small deep Pls are associated with sensory symptoms and eye movement disorders. ${ }^{3}$ However, the reason for this difference in $\mathrm{PI}$ location and the factors affect- 
ing it have not been clearly verified.

Hypertension was previously considered a major factor affecting deep PI. ${ }^{2}$ On the other hand, paramedian Pls were associated with ectasia of the basilar artery (BA), which may stretch and distort the orifices of the paramedian perforators or alter the blood flow that forms in areas prone to early atherosclerosis that is invisible on conventional neuroimaging. ${ }^{4}$ The presence of a BA plaque in patients without a significant BA stenosis on high-resolution magnetic resonance imaging (HR-MRI) was more closely associated with paramedian Pls than deep PIs. ${ }^{5} \mathrm{HR}-\mathrm{MRI}$ is sensitive for detecting early atherosclerotic changes, and the location of these early atherosclerotic plaques is likely associated with artery shape, which influences the hemodynamic properties and atherosclerosis development. ${ }^{6}$

The posterior circulation has a greater degree of geometric variation than the anterior circulation. The vascular geometry of the posterior circulation may include dominance of the vertebral arteries (VAs), course of the BA, and angles of the VAs and BA. PI type and vertical location may be influenced by the location of the BA plaque, which is likely associated with the vascular geometry, especially the angles of the vertebrobasilar arteries. Here we hypothesized that the angles of the vertebrobasilar system may affect the BA plaque location in isolated PI patients, whereas the BA plaque location may differ by PI type and vertical location. Patients without significant BA stenosis, rather than those with significant stenosis, would be optimal subjects for exploring the relationship between vertebrobasilar system angles and the early atherosclerotic changes influencing PI location.

\section{Methods}

\section{Participants}

Patients with a PI within 7 days of stroke onset, who were admitted to the stroke center of Kyung Hee University Hospital between July 2011 and March 2016, were screened. Among them, patients without BA stenosis from the time-of-flight magnetic resonance angiography (TOF-MRA) were consecutively enrolled. PI presence and location were confirmed using diffusion-weighted imaging (DWI) and fluid-attenuated inversion recovery (FLAIR) images taken on the day of admission. At the same time, TOF-MRA was performed and all patients with an isolated PI without BA stenosis underwent an additional HR-MRI based on the DWI and TOF-MRA findings. Patients with (1) an embolic source in the proximal artery or heart and (2) those showing poor image quality were excluded. Stroke severity and functional outcome at discharge were measured using National Institute of Health Stroke Scale (NIHSS) score and modified Rankin Scale (mRS) score. Institutional Review Board of Kyung Hee University
Hospital (KMC IRB 2016-06-201) approved this study but waived the need for informed consent because of its retrospective nature and minimal risk to patients.

\section{Imaging acquisition}

MRI was performed using a 3.0 T Philips scanner (Philips Healthcare, Eindhoven, the Netherlands) with a standard 16-channel neurovascular coil on the day of admission. The sequences included DWI, FLAIR, enhanced T1 weighted image, gradient echo image, TOF-MRA, and proton density black blood image (PD$\mathrm{BBI}$ ). PD-BBI was obtained using the following parameters along the $B A$ axis: relaxation time/echo time $=2,500 / 30 \mathrm{~ms}$, matrix $=320 \times 220$, field-of-view $=120 \times 110 \mathrm{~mm}$, slice thickness $/$ gap $=2 / 0 \mathrm{~mm}$, longitudinal coverage $=60 \mathrm{~mm}$ (30 slices), actual voxel size $=0.375 \times 0.5 \times 2 \mathrm{~mm}$, and reconstructed voxel size $=0.23 \times 0.23 \times 2 \mathrm{~mm}$. The pre-regional $80-\mathrm{mm}$-thick saturation pulse was used to saturate the incoming arterial flow.

\section{$\mathrm{PI}$ and basilar plaque}

PI location was classified by type and vertical location (Figure 1). PI type was classified as (1) paramedian PI (when the ischemic lesion abutted the base of the pons); (2) deep PI (when the ischemic lesion did not reach the base of the pons); or (3) lateral PI (when the lesion was located lateral to the pons). Vertically, the PI was classified as: (1) rostral (at the level of a relatively round shape with a small round aqueduct); (2) middle (at the level of a square fourth ventricle, large middle cerebellar peduncles, and silhouettes of the trigeminal nerves); and (3) caudal (at the level of a similarly shaped pons to the middle pons level but with images of the facial/acoustic nerves and grooves instead of the trigeminal nerves) PI according to the
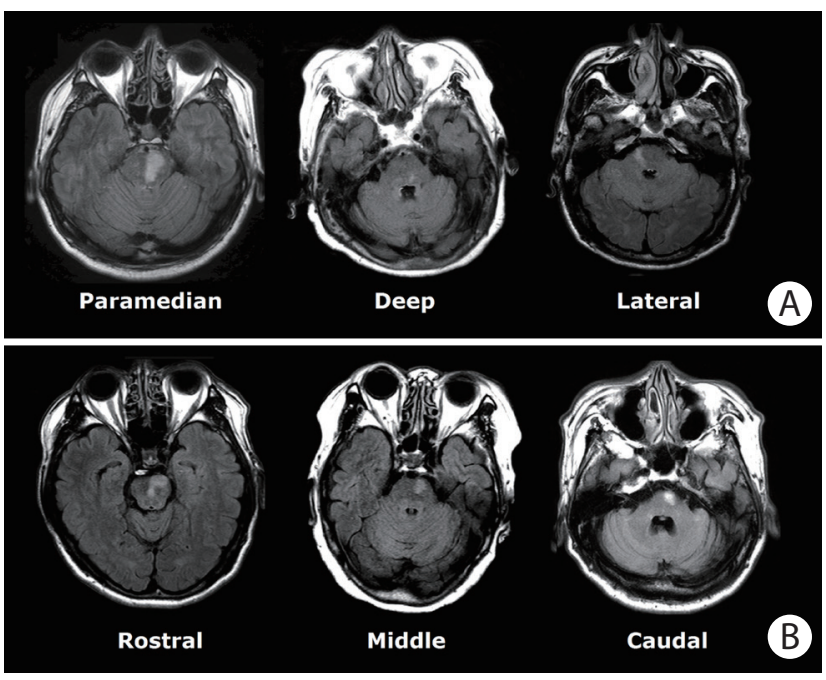

Figure 1. Classification of pontine infarction by types (A) and vertical locations (B). 

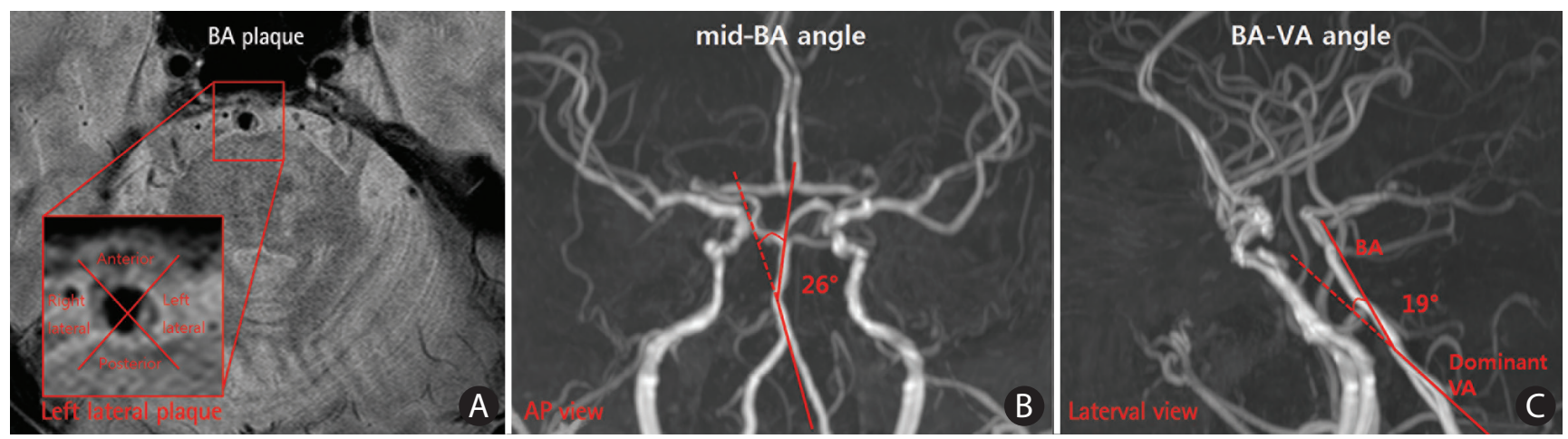

Figure 2. BA plaque location (A) and the measurement of mid-BA (B) and BA-VA angles (C). BA, basilar artery; VA, vertebral artery.

shape of the pons and the fourth ventricle. ${ }^{2}$

$\mathrm{BA}$ plaque presence, location, and length were assessed on the PD-BBI. A focal and eccentric wall thickening on the PD$\mathrm{BBI}$ of $\mathrm{BA}$ was defined as a BA plaque. ${ }^{5}$ The culprit plaque for the PI was defined as the plaque observed inside the BA from the identical axial slice where the PI was observed. The BA was divided into four quadrants to determine the plaque location (Figure 2A): anterior, posterior, or lateral (right and left). If the plaque was observed in more than two quadrants, the quadrant with the maximal BA plaque was chosen. Since the slice thickness of the MRI protocol was $2 \mathrm{~mm}$, the plaque length was calculated as being twice the number of axial slices with a visible basilar plaque culprit for the PI.

\section{Vertebrobasilar artery angles}

The VA and BA angles were measured at the anteroposterior (AP) view and the lateral view of the 3D-reconstructed TOFMRA (Figure 2). The VA dominance and mid-BA angle were determined and measured in the AP view. The angle between the dominant VA and BA was measured in the lateral view. The dominant VA was defined as the VA with a larger diameter or connected to the BA with a straighter manner.? Imaginary lines were drawn from the mid-BA to the vertebrobasilar junction and the top of the BA in the AP view (Figure 2B). The maximum angle between these two imaginary lines was considered the mid-BA angle. In the same manner, imaginary lines were drawn from the vertebrobasilar junction to the $B A$ and the dominant $V A$, whereas the angle between the two lines was considered the lateral BA-VA angle (Figure 2C).

\section{Statistical analysis}

First, demographics, risk factors for atherosclerosis, and vascular geometry were compared between patients with and those without BA plaques. Multivariate analysis was performed to explore the independent factors associated with the presence of BA plaques. Demographic data, conventional risk factors for atherosclerosis, and angles of the vertebrobasilar arteries were selected for entry into the model. Second, demographics, risk factors, and vascular geometry were compared among patients with paramedian, deep, and lateral Pls. Third, the identical comparison was performed among patients with rostral, middle, and caudal Pls. The chi-square test, Fisher exact test, oneway analysis of variance, or Student t-test was appropriately used to compare the variables. $P$-values $<0.05$ were considered statistically significant. SPSS version 18 (SPSS Inc., Chicago, IL, USA) was used for the statistical analysis.

\section{Results}

During the study period, 102 patients with isolated Pls who underwent HR-MRI were enrolled. Six patients were excluded (two with atrial fibrillation and four with significant stenosis at the proximal VA); ultimately, 96 were included in the analysis. The mean age was $69 \pm 11$ years, and 49 patients $(51 \%)$ were male. Pls were classified as paramedian in $70(72.9 \%)$, deep in $17(17.7 \%)$, and lateral in nine patients (9.4\%). Fifty-one patients (53.1\%) had a right $\mathrm{PI}, 44$ (45.8\%) had a left PI, and one patient (1.0\%) had bilateral PI. Vertically, the Pls were located at the rostral $(32.3 \%, n=31)$, middle $(42.7 \%, n=41)$, and caudal $(25.0 \%, n=24)$ regions.

BA plaques were observed in 64 patients (66.7\%) on HR-MRI. There was no difference in risk factors between patients with and those without a BA plaque (Table 1). Plaques were more frequently observed in patients with paramedian or lateral Pls than those with deep Pls $(72.9 \%$ or $77.8 \%$ vs. $35.3 \%$, respectively; $P=0.01$ ). Patients with a BA plaque showed a higher discharge NIHSS score (4 [interquartile range (IQR), 3 to 5] vs. 2 [IQR, 1 to 4], $P=0.006$ ) and $m R S$ score (2 [IQR, 2 to 3] vs. 1 [IQR, 1 to 3], $P=0.007)$ than those without a plaque. The BA-VA angle was greater in patients with a $\mathrm{BA}$ plaque than in those without 
Table 1. Baseline characteristics of patients with and without basilar artery plaque

\begin{tabular}{|c|c|c|c|}
\hline & $\begin{array}{l}\text { Plaque }(+) \\
(n=64)\end{array}$ & $\begin{array}{c}\text { Plaque (-) } \\
(n=32)\end{array}$ & $P$ \\
\hline Age (yr) & $71 \pm 10$ & $65 \pm 11$ & 0.32 \\
\hline Male sex & $32(50.0)$ & $15(46.9)$ & 0.83 \\
\hline Hypertension & $48(75.0)$ & $23(71.9)$ & 0.81 \\
\hline Diabetes & $24(37.5)$ & 14 (43.8) & 0.66 \\
\hline Hyperlipidemia & $44(68.8)$ & $21(65.6)$ & 0.82 \\
\hline Smoking & $22(34.4)$ & $11(34.4)$ & 1.00 \\
\hline \multicolumn{4}{|l|}{ Type of pontine infarction } \\
\hline Paramedian & $51(79.7)$ & 19 (59.4) & 0.01 \\
\hline Deep & $6(9.4)$ & $11(34.4)$ & \\
\hline Lateral & $7(10.9)$ & $2(6.2)$ & \\
\hline \multicolumn{4}{|l|}{ Vertical location } \\
\hline Rostral & $16(25.0)$ & $15(46.9)$ & 0.09 \\
\hline Middle & $31(48.4)$ & 10 (31.2) & \\
\hline Caudal & $17(26.5)$ & $7(21.9)$ & \\
\hline Discharge NIHSS score & $4(3-5)$ & $2(1-4)$ & 0.006 \\
\hline Discharge $\mathrm{mRS}$ score & $2(2-3)$ & $1(1-3)$ & 0.007 \\
\hline \multicolumn{4}{|c|}{ Angles of vertebrobasilar arteries } \\
\hline BA-VA angle $\left({ }^{\circ}\right)$ & $35 \pm 13$ & $23 \pm 13$ & $<0.001$ \\
\hline Mid-BA angle ( $\left.{ }^{\circ}\right)$ & $25 \pm 17$ & $23 \pm 19$ & 0.68 \\
\hline
\end{tabular}

Values are presented as mean \pm standard deviation, number $(\%)$, or median (interquartile range).

NIHSS, National Institute of Health Stroke Scale; mRS, modified Rankin

Scale; BA, basilar artery; VA, vertebral artery. $\left(35^{\circ} \pm 13^{\circ}\right.$ vs. $\left.23^{\circ} \pm 13^{\circ}, P<0.001\right)$ (Table 1). After adjusting for covariates, old age (odds ratio [OR], $1.071 ; 95 \% \mathrm{Cl}, 1.020$ to 1.123 ; $P=0.006)$ and a large $B A-V A$ angle $(O R, 1.077 ; 95 \% \mathrm{Cl}, 1.033$ to $1.124 ; P=0.001)$ were independently associated with the presence of a BA plaque.

\section{Plaque location by PI type}

BA plaques were most frequently located at the posterior wall $(46.9 \%, n=30)$, followed by the lateral $(32.8 \%, n=21)$ and anterior $(20.3 \%, n=13)$ walls of the BA. Of the 21 lateral wall plaques, 15 were located at the ipsi-lesional side of the PI, whereas six were located at the contra-lesional side.

There was no difference in risk factors by PI types (Table 2). BA plaque location differed by $\mathrm{PI}$ types $(P=0.03)$; posterior wall plaques were more frequent in patients with paramedian PIs than in those with deep or lateral Pls (37.1\% vs. $11.8 \%$ or $22.2 \%$, respectively). Lateral wall plaques were more frequent in patients with lateral Pls than in those with paramedian or deep Pls (55.5\% vs. $20.0 \%$ or $11.8 \%$, respectively). The mid$\mathrm{BA}$ angle also differed by PI types $(P=0.009)$; patients with a lateral $\mathrm{PI}$ had a greater mid-BA angle than those with a paramedian or deep PI $\left(36^{\circ} \pm 23^{\circ}\right.$ vs. $21^{\circ} \pm 16^{\circ}$ or $31^{\circ} \pm 18^{\circ}$, respectively).

Table 2. Comparison of variables by pontine infarction type

\begin{tabular}{|c|c|c|c|c|}
\hline & \multicolumn{3}{|c|}{ Pontine infarction type } & \multirow{2}{*}{$P$} \\
\hline & Paramedian $(n=70)$ & Deep $(n=17)$ & Lateral $(n=9)$ & \\
\hline Age (yr) & $70 \pm 11$ & $70 \pm 8$ & $64 \pm 13$ & 0.33 \\
\hline Male sex & $32(45.7)$ & $9(52.9)$ & $6(66.7)$ & 0.47 \\
\hline Hypertension & $52(74.3)$ & $12(70.6)$ & $7(77.8)$ & 0.92 \\
\hline Diabetes & $29(41.4)$ & $6(35.3)$ & $3(33.3)$ & 0.83 \\
\hline Hyperlipidemia & $50(71.4)$ & $8(47.1)$ & $7(77.8)$ & 0.12 \\
\hline Current smoking & $22(31.4)$ & $7(41.2)$ & $4(44.4)$ & 0.60 \\
\hline Location & & & & 0.03 \\
\hline No plaque & $19(27.1)$ & $11(64.7)$ & $2(22.2)$ & \\
\hline Anterior & $11(15.7)$ & $2(11.8)$ & 0 & \\
\hline Posterior & $26(37.1)$ & $2(11.8)$ & $2(22.2)$ & \\
\hline Lateral & $14(20.1)$ & $2(11.7)$ & $5(55.6)$ & \\
\hline Plaque length (mm) & $2.0(1.6)$ & $0.9(1.5)$ & $2.0(2.5)$ & 0.05 \\
\hline Discharge NIHSS score & $4(2-5)$ & $1(0.5-3.5)$ & $3(2-3.5)$ & 0.01 \\
\hline Discharge mRS score & $2(1-3)$ & $1(1-3)$ & $2(2-3)$ & 0.10 \\
\hline \multicolumn{5}{|c|}{ Angles of vertebrobasilar arteries } \\
\hline BA-VA angle $\left({ }^{\circ}\right)$ & $31 \pm 15$ & $26 \pm 10$ & $39 \pm 10$ & 0.07 \\
\hline Mid-BA angle $\left({ }^{\circ}\right)$ & $21 \pm 16$ & $31 \pm 18$ & $36 \pm 23$ & 0.009 \\
\hline
\end{tabular}

Values are presented as mean \pm standard deviation, number (\%), or median (interquartile range).

NIHSS, National Institute of Health Stroke Scale; mRS, modified Rankin Scale; BA, basilar artery; VA, vertebral artery. 
Table 3. Comparison of variables by pontine infarction vertical location

\begin{tabular}{|c|c|c|c|c|}
\hline & \multicolumn{3}{|c|}{ Vertical location of pontine infarction } & \multirow{2}{*}{$P$} \\
\hline & Rostral $(n=31)$ & Middle $(n=41)$ & Caudal $(n=24)$ & \\
\hline Age (yr) & $68 \pm 13$ & $71 \pm 9$ & $68 \pm 9$ & 0.43 \\
\hline Male sex & $15(48.4)$ & $21(51.2)$ & $11(45.8)$ & 0.91 \\
\hline Hypertension & $21(67.7)$ & $31(75.6)$ & $19(79.2)$ & 0.60 \\
\hline Diabetes & $12(38.7)$ & $16(39.0)$ & $10(41.7)$ & 0.97 \\
\hline Hyperlipidemia & $21(67.7)$ & $26(63.4)$ & $18(75.5)$ & 0.63 \\
\hline Current smoking & $10(32.3)$ & $15(36.6)$ & $8(33.3)$ & 0.92 \\
\hline Location & & & & $<0.001$ \\
\hline No plaque & $15(48.4)$ & $10(24.4)$ & $7(29.2)$ & \\
\hline Anterior & 0 & $11(26.8)$ & $2(8.3)$ & \\
\hline Posterior & $10(32.3)$ & $6(14.6)$ & $14(58.3)$ & \\
\hline Lateral & $6(19.3)$ & $14(34.2)$ & $1(4.2)$ & \\
\hline Plaque length $(\mathrm{mm})$ & $1.4(1.5)$ & $2.2(1.9)$ & $1.7(1.5)$ & 0.11 \\
\hline Discharge NIHSS score & $3(1-4)$ & $4(1.5-4)$ & $3(2-5)$ & 0.43 \\
\hline Discharge $\mathrm{mRS}$ score & $2(1-3)$ & $2(1-3)$ & $3(1-3)$ & 0.44 \\
\hline \multicolumn{5}{|c|}{ Angles of vertebrobasilar arteries } \\
\hline BA-VA angle ( $\left.{ }^{\circ}\right)$ & $28 \pm 13$ & $27 \pm 13$ & $41 \pm 13$ & $<0.001$ \\
\hline Mid-BA angle ( $\left.{ }^{\circ}\right)$ & $21 \pm 19$ & $29 \pm 18$ & $18 \pm 13$ & 0.03 \\
\hline
\end{tabular}

Values are presented as mean \pm standard deviation, number (\%), or median (interquartile range).

NIHSS, National Institutes of Health Stroke Scale; mRS, modified Rankin Scale; BA, basilar artery; VA, vertebral artery.

Table 4. Comparison of variables by basilar artery plaque location

\begin{tabular}{|c|c|c|c|c|c|}
\hline & None & Anterior & Posterior & Lateral & $P$ \\
\hline Age (yr) & $65 \pm 11$ & $70 \pm 9$ & $72 \pm 9$ & $72 \pm 11$ & 0.02 \\
\hline Male sex & $13(44.8)$ & $7(53.8)$ & $18(54.5)$ & $9(42.9)$ & 0.79 \\
\hline Hypertension & $23(71.9)$ & $11(84.6)$ & $20(66.7)$ & 17 (81.0) & 0.58 \\
\hline Diabetes & $14(43.8)$ & $4(30.8)$ & $13(43.3)$ & 7 (33.3) & 0.73 \\
\hline Hyperlipidemia & $21(65.6)$ & $7(53.8)$ & $22(73.3)$ & 15 (71.4) & 0.44 \\
\hline Smoking & $11(34.4)$ & $6(46.2)$ & $11(36.7)$ & $5(23.8)$ & 0.59 \\
\hline \multicolumn{6}{|c|}{ Angles of vertebrobasilar arteries } \\
\hline BA-VA angle $\left({ }^{\circ}\right)$ & $23 \pm 13$ & $32 \pm 14$ & $38 \pm 14$ & $31 \pm 11$ & 0.004 \\
\hline Mid-BA angle $\left({ }^{\circ}\right)$ & $23 \pm 19$ & $23 \pm 13$ & $19 \pm 16$ & $34 \pm 18$ & 0.03 \\
\hline Plaque length $(\mathrm{mm})$ & NA & $3.2 \pm 1.7$ & $2.6 \pm 1.1$ & $2.5 \pm 1.6$ & $0.26^{*}$ \\
\hline
\end{tabular}

Values are presented as mean \pm standard deviation or number (\%)

$\mathrm{BA}$, basilar artery; $\mathrm{VA}$, vertebral artery; NA, not available.

*Plaque length was compared among those with a plaque.

\section{Plaque location by PI vertical location}

There was no difference in risk factors by PI vertical locations (Table 3). However, the BA plaque location differed by PI vertical location $(P<0.001)$; BA plaques were observed in a high proportion of patients with middle $(75.6 \%)$ and caudal $(70.8 \%)$ Pls. BA plaques were frequently located at the lateral $(34.1 \%, n=14)$ and anterior $(26.5 \%, n=11)$ wall in patients with middle Pls and at the posterior wall $(58.3 \%, n=14)$ in patients with caudal Pls. Only $51.6 \%$ of pa- tients with a rostral PI had a BA plaque (Table 3).

The BA-VA and mid-BA angles also differed by BA plaque vertical locations; the BA-VA angle was greater in patients with caudal Pls than in those with rostral or middle PIs $\left(41^{\circ} \pm 13^{\circ}\right.$ vs. $28^{\circ} \pm 13^{\circ}$ or $27^{\circ} \pm 13^{\circ}$, respectively; $\left.P<0.001\right)$. The mid-BA angle was greater in patients with middle Pls than in those with rostral or caudal Pls $\left(29^{\circ} \pm 18^{\circ}\right.$ vs. $21^{\circ} \pm 19^{\circ}$ or $18^{\circ} \pm 13^{\circ}$, respectively; $P<0.001$ ). 


\section{Plaque location and vertebrobasilar artery angles}

There was no difference in risk factors except age among patients with different BA plaque locations (Table 4). However, the BA-VA angle differed by BA plaque location; the BA-VA angle was greater in patients with posterior plaques than in those without a plaque or those with anterior or lateral plaques $\left(38^{\circ} \pm 14^{\circ}\right.$ vs. $23^{\circ} \pm 13^{\circ}$, $32^{\circ} \pm 14^{\circ}, 31^{\circ} \pm 11^{\circ}$, respectively; $\left.P=0.004\right)$. The mid-BA angle differed by $B A$ plaque locations; the mid-BA angle was greater in patients with lateral plaques than in patients without a plaque or those with anterior or posterior plaques $\left(34^{\circ} \pm 18^{\circ}\right.$ vs. $23^{\circ} \pm 19^{\circ}$, $23^{\circ} \pm 13^{\circ}$ or $19^{\circ} \pm 16^{\circ}$, respectively; $P=0.03$ ).

\section{Discussion}

In the present study, vertebrobasilar system angles were associated with BA plaque location, which differed according to PI types and vertical locations. BA plaques were less frequently observed in patients with a deep PI by type and a rostral PI by vertical location. High angulation of the mid-BA on the AP view was associated with lateral $B A$ plaques, lateral $\mathrm{PI}$ with type, and middle PI by vertical location. A greater angle between the dominant VA and BA on the lateral view was associated with a posterior BA plaque, with paramedian PI by type, and with lower PI by vertical location.

Vascular geometry of the parental artery affects the hemodynamics inside the vasculature and likely determines preclinical atherosclerosis location. ${ }^{6}$ Furthermore, the angulation of the parental artery might be associated with infarction presence and location. ${ }^{78}$ In the vertebrobasilar system, turbulence occurs at the vertebrobasilar junction, where the two VA flows conjoin, and at the mid-BA when the flow bends because of the mid-BA angle. ${ }^{910}$ However, the blood flow recovers to laminar flow at the distal BA, which may partially explain the low incidence of BA plaques in the rostral PI compared to middle and caudal $\mathrm{Pls}^{9}$ (Supplementary Figure 1). According to our study, patients with a lateral plaque demonstrated the highest mid-BA angle from the AP view and patients with a posterior plaque demonstrated the largest BA-VA angle from the lateral view. Furthermore, the mid-BA angle from the AP view was greater in patients with middle and lateral Pls, whereas the BA-VA angle from the lateral view was greater in patients with caudal Pls. Therefore, vertebrobasilar artery angle may influence BA plaque development and location.

The presence of a BA plaque in those with an isolated PI was $66.7 \%$, a finding that is compatible with the previous report. ${ }^{5}$ In our study, BA plaque location differed by PI types and vertical locations. The majority of deep Pls showed no BA plaques, which suggests that small vessel disease in the perforators is a dominant stroke mechanism in deep PI. ${ }^{11}$ On the other hand, early atherosclerotic changes in a BA might be the dominant mechanism in cases of paramedian or lateral Pls. Patients with lateral Pls showed more plaques at the lateral wall of the BA, where the lateral circumferential branches of BA perforators originate. ${ }^{12}$ Likewise, paramedian infarctions were more likely to have plaques at the posterior BA wall, where the paramedian perforator branches originate. By vertical location, lateral BA plaques were more frequently seen in middle Pls, whereas posterior wall BA plaques were more frequently seen in caudal Pls. Patients with a posterior circulation infarction due to a significant BA stenosis previously showed predominance in BA plaque location at the anterior wall of the BA. ${ }^{13}$ In contrast, our study demonstrated that BA plaques in isolated Pls without a BA stenosis were more frequently located at the posterior wall. Since isolated PIs without a BA stenosis are directly caused by obliteration of the perforators, which usually arise from the posterior aspect of the BA, the predominance in plaque location corresponds well with the pathophysiology of isolated Pls without BA stenosis.

Our study has several noteworthy limitations. First, the sample size was small and only a single center was included. Second, the angle was measured from the $2 \mathrm{D}$ plane of a 3D-reconstructed TOF-MRA (AP or lateral view). However, this method is considered valid for accurately measuring cerebral artery geometry. ${ }^{14,15}$ Third, PD-BBI was used to evaluate BA plaques; therefore, the composition of the BA plaques could not be assessed. Finally, we measured the vascular geometry but could not evaluate the true hemodynamics that might explain the association between vascular geometry and atherosclerotic plaque seen on HR-MRI. Further studies measuring the hemodynamic factors may elucidate the exact hemodynamic mechanism linking arterial angles to BA plaque and PI locations.

\section{Conclusions}

In conclusion, a greater angulation between the dominant VA and BA from the lateral view may enhance BA plaque formation on the posterior wall at the lower pontine level and cause paramedian or caudal Pls. A greater angulation at the mid-BA from the AP view may enhance BA plaque formation on the lateral wall at the middle pontine level and cause a lateral or middle PI. A BA plaque at a specific location, determined using vascular geometry, obliterates the orifice of the perforators and affects PI type and location. 


\section{Supplementary materials}

Supplementary materials related to this article can be found online at https://doi.org/10.5853/jos.2017.00829.

\section{Disclosure}

The authors have no financial conflicts of interest.

\section{Acknowledgments}

This work was supported by a grant from Kyung Hee University in 2017 (KHU-20170850).

\section{References}

1. Bassetti $C$, Bogousslavsky J, Barth $A$, Regli F. Isolated infarcts of the pons. Neurology 1996;46:165-175.

2. Kim JS, Lee JH, Im JH, Lee MC. Syndromes of pontine base infarction. A clinical-radiological correlation study. Stroke 1995;26:950-955.

3. Kumral E, Bayülkem G, Evyapan D. Clinical spectrum of pontine infarction. Clinical-MRI correlations. J Neurol 2002;249:16591670.

4. Kwon HM, Kim JH, Lim JS, Park JH, Lee SH, Lee YS. Basilar artery dolichoectasia is associated with paramedian pontine infarction. Cerebrovasc Dis 2009;27:114-118.

5. Klein IF, Lavallée PC, Mazighi M, Schouman-Claeys $E_{1}$ Labreuche J, Amarenco P. Basilar artery atherosclerotic plaques in paramedian and lacunar pontine infarctions: a high-resolution MRI study. Stroke 2010;41:1405-1409.

6. Kim BJ, Yoon Y, Lee DH, Kang DW, Kwon SU, Kim JS. The shape of middle cerebral artery and plaque location: high-resolution MRI finding. Int J Stroke 2015;10:856-860.

7. Hong JM, Chung CS, Bang OY, Yong SW, Joo IS, Huh K. Vertebral artery dominance contributes to basilar artery curvature and peri-vertebrobasilar junctional infarcts. J Neurol Neurosurg Psychiatry 2009;80:1087-1092.

8. Jeong SK, Lee JH, Nam DH, Kim JT, Ha YS, Oh SY, et al. Basilar artery angulation in association with aging and pontine lacunar infarction: a multicenter observational study. J Atheroscler Thromb 2015;22:509-517.

9. Ravensbergen J, Krijger JK, Hillen B, Hoogstraten HW. The influence of the angle of confluence on the flow in a vertebrobasilar junction model. J Biomech 1996;29:281-299.

10. Wake-Buck AK, Gatenby JC, Gore JC. Hemodynamic characteristics of the vertebrobasilar system analyzed using MRIbased models. PLoS One 2012;7:e51346.

11. Caplan LR. Lacunar infarction and small vessel disease: pathology and pathophysiology. J Stroke 2015;17:2-6.

12. Lescher $\mathrm{S}$, Samaan T, Berkefeld J. Evaluation of the pontine perforators of the basilar artery using digital subtraction angiography in high resolution and $3 \mathrm{D}$ rotation technique. AJNR Am J Neuroradiol 2014;35:1942-1947.

13. Huang B, Yang WQ, Liu XT, Liu HJ, Li PJ, Lu HK. Basilar artery atherosclerotic plaques distribution in symptomatic patients: a 3.0T high-resolution MRI study. Eur J Radio/ 2013;82:e199e203.

14. Kim BJ, Kim SM, Kang DW, Kwon SU, Suh DC, Kim JS. Vascular tortuosity may be related to intracranial artery atherosclerosis. Int J Stroke 2015;10:1081-1086.

15. Kim BJ, Kim SM, Ahn SH, Kang DW, Kwon SU, Kim JS. Lateral thalamic infarction and the vascular geometry of the posterior cerebral artery. Cerebrovasc Dis 2016;41:8-12. 


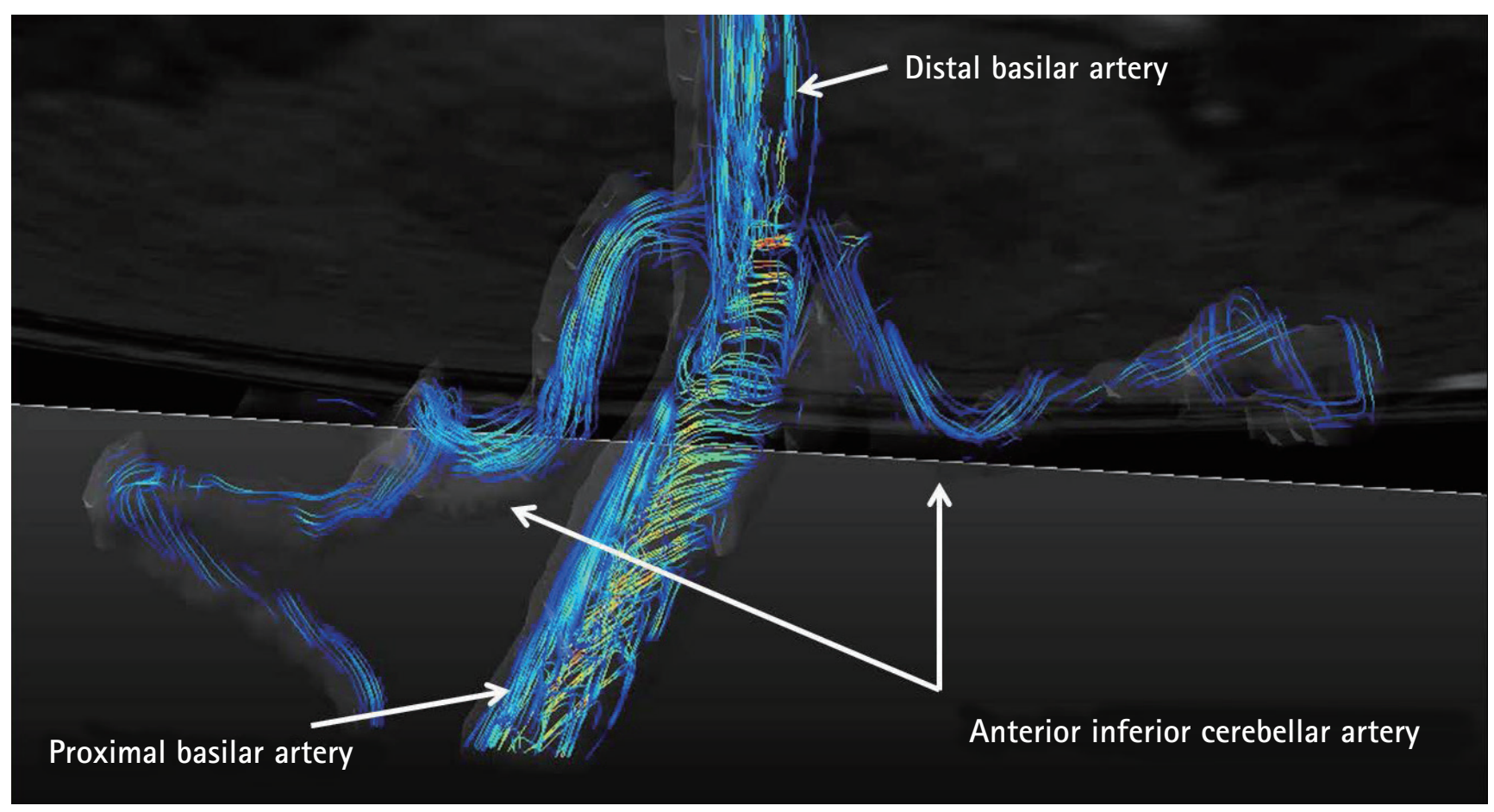

Supplementary Figure 1. Real-time flow measurement of a patient with rostral pontine infarction. Flow pattern of distal basilar artery recovers to laminar flow pattern, whereas turbulence is observed from the middle and proximal basilar artery. 